\title{
West Syndrome
}

National Cancer Institute

\section{Source}

National Cancer Institute. West Syndrome. NCI Thesaurus. Code C84788.

A rare autosomal recessive inherited neurodegenerative disorder caused by mutations in the PLA2G6 gene. It is characterized by the development of swellings called spheroids along the axons of the central nervous system. Signs and symptoms appear early in life and include movement difficulties, muscle hypotonia and spasticity, and cog nitive dysfunction. 